\title{
Beneficial role of microRNA-328-3p in fracture healing by enhancing osteoblastic viability through the PTEN/PI3K/AKT pathway
}

\author{
WEI XIE, ZHUQING WANG, YANBO ZHANG and ZHENPENG ZHANG
}

Department of Sport Medicine, Traditional Chinese Medicine Hospital of Weifang, Weifang, Shandong 261041, P.R. China

Received June 9, 2020; Accepted September 29, 2020

DOI: $10.3892 /$ etm.2020.9401

\begin{abstract}
Although fragility fracture is a global public health burden, the mechanisms underlying fracture healing remain unclear. The present study aimed to assess the dynamic expression pattern of microRNA-328-3p (miR-328-3p) during fracture healing in patients with fragility fracture and to explore the functional role and mechanisms of miR-328-3p in the regulation of osteoblastic viability. The expression levels of miR-328-3p was examined using reverse transcription-quantitative PCR (RT-qPCR). Osteoblastic proliferation and apoptosis were analyzed via MTT and flow cytometry assays. A luciferase reporter assay was adopted to confirm the interaction between miR-328-3p and its target gene PTEN, and western blotting was used to explore the activity of PI3K/AKT signaling. The results of the present study demonstrated that serum miR-328-3p expression did not significantly differ at the early stage of healing in patients with fracture, but was markedly decreased 14 and 21 days post fixation $(\mathrm{P}<0.01)$. PTEN was demonstrated to be a target gene of miR-328-3p and was inhibited by miR-328-3p overexpression in osteoblasts $(\mathrm{P}<0.001)$. miR-328-3p overexpression increased osteoblastic proliferation but decreased apoptotic rate, with these effects being reversed by PTEN overexpression $(\mathrm{P}<0.05)$. The expression of phosphorylated-AKT was elevated in osteoblasts by miR-328-3p overexpression, but this effect was abolished by overexpressing PTEN. Thus, the present study revealed that miR-328-3p may accelerate fracture healing by promoting osteoblastic viability through the PTEN/PI3K/AKT pathway.
\end{abstract}

\section{Introduction}

Fragility fracture generally occurs due to an injury that would be insufficient to cause fracture in a normal bone (1). It is a

Correspondence to: Dr Zhenpeng Zhang, Department of Sport Medicine, Traditional Chinese Medicine Hospital of Weifang, 1055 Weizhou Road, Weifang, Shandong 261041, P.R. China

E-mail: yunxiang0222@163.com

Key words: fracture healing, microRNA-328-3p, osteoblast, proliferation, apoptosis, PTEN, PI3K/AKT signaling common disease in the elderly, leading to huge burdens on health and the economy, worldwide (2). Fragility fracture has become a global public health threat, and 2,000,000 new cases occur in the USA annually (3). In general, fractures lead to acute pain, decreased mobility and loss of independence, and almost all patients require long-term fixation (4). However, fracture healing is a slow recovery process, leading to a significant reduction in patient quality of life (5). Thus, the mechanisms underlying fracture healing urgently need to be explored further, which may help to reduce the time for fracture healing. Fracture healing involves multiple growth factors and cytokines that are associated with the proliferation, apoptosis and differentiation of osteoblasts (6). To accelerate bone regeneration, strategies to facilitate osteoblastic activation are necessary.

MicroRNAs (miRNAs) are a group of small non-coding RNAs with important regulatory roles in various cellular processes, such as cell proliferation, migration, invasion, differentiation and apoptosis (7). They can directly bind the 3'-unstranlated region (3'-UTR) of target messenger RNAs (mRNAs), leading to the inhibition in target gene expression at post-transcriptional levels (8). Emerging studies have reported the roles of miRNAs in the regulation of osteoblastic activity. For example, Zhang et al (9) observed that miR-146a suppressed osteoblastic proliferation and enhanced apoptosis. Li et al (10) revealed that miR-342-5p could inhibit osteoblastic cell proliferation, migration and differentiation by targeting Bmp7. Differentially expressed miR-125a-3p in the osteoblastic differentiation process could significantly regulate osteoblastic cell proliferation (11). The decreased expression levels of miR-491-3p in postmenopausal osteoporosis regulates osteoblastic viability and differentiation (12). miR-328-3p has also been demonstrated to regulate the proliferation of different types of cell, such as colorectal cancer (13) and osteosarcoma cells (14). Weilner et al (15) investigated the differentially expressed circulating miRNAs that may be associated with osteoporotic fracture, the results of which revealed that miR-328-3p was associated with osteogenic differentiation, and was downregulated in patients with osteoporosis who developed fractures. However, whether miR-328-3p is involved in fracture healing processes remains unclear.

The present study assessed the expression of miR-328-3p during healing in patients with fragility fracture and the potential regulatory effect of miR-328-3p on osteoblastic 
viability. In addition, the target gene of miR-328-3p was also predicted and explored, which may provide a novel insight into the pathogenesis of fracture healing.

\section{Materials and methods}

Patients and sample collection. The present study included 80 patients with fragility fracture (34 males, 46 females; average age, $68.94 \pm 11.30$ years; age range, $48-87$ years), who underwent fixation therapy at the Traditional Chinese Medicine Hospital of Weifang from January 2016 to December 2018. All patients suffered from fragility fracture without trauma or due to low-energy trauma. Before fixation therapy, blood samples were collected from the patients within $24 \mathrm{~h}$ of the fracture. After fixation, blood samples were obtained on day 7,14 and 21. In addition, the blood samples of 40 healthy volunteers were also collected during the same time period. All blood samples were immediately centrifuged at $1,500 \mathrm{x}$ g for $10 \mathrm{~min}$ at $4^{\circ} \mathrm{C}$ for serum extraction and stored at $-80^{\circ} \mathrm{C}$ for subsequent use. The healthy individuals included in the current study comprised 19 males and 21 females, with an average age of $68.10 \pm 12.05$ years (range of 46-86 years). All the participants signed written informed consent for clinical sample collection and analysis, and the experimental procedures were approved by the Ethics Committee of Traditional Chinese Medicine Hospital of Weifang.

Cell culture and transfection. A human osteoblast cell line hFOB1.19 was purchased from the Chinese Academy of Sciences Cell Bank. The cells were cultured in RPMI-1640 (HyClone; Cytiva) medium supplemented with 10\% FBS (Invitrogen; Thermo Fisher Scientific, Inc.), 1,000 U/ml penicillin and $100 \mu \mathrm{g} / \mathrm{ml}$ streptomycin in an incubator with $5 \% \mathrm{CO}_{2}$ at $37^{\circ} \mathrm{C}$.

To overexpress miR-328-3p in hFOB1.19, $50 \mathrm{nM}$ miR-328-3p mimic (5'-CUGGCCCUCUCUGCCCUUCCG U-3') was synthesized by Shanghai GenePharma Co., Ltd. and transfected into cells using Lipofectamine 3000 (Invitrogen; Thermo Fisher Scientific, Inc.) following the manufacturer's instructions. The negative control for the mimic (mimic NC; $50 \mathrm{nM}$; Shanghai GenePharma Co., Ltd.; 5'-UUCUCCGAA CGUGUCACGU-3') was used and transfected into hFOB1.19 to serve as a negative control group. Additionally, the overexpression vector of PTEN, pcDNA3.1-PTEN (Shanghai GenePharma Co., Ltd.), was synthesized and transfected into hFOB1.19 to overexpress PTEN in osteoblasts. The cells transfected with only transfection reagent were set as the mock group. Cell transfection was performed at $37^{\circ} \mathrm{C}$ for $6 \mathrm{~h}$, the transfection regent was removed and fresh RPMI-1640 medium was added. After $48 \mathrm{~h}$ of cell transfection, cells were used for subsequent experimentation. All experiments were repeated in triplicate.

$R N A$ extraction and reverse transcription-quantitative PCR $(R T-q P C R)$. TRIzol ${ }^{\circledR}$ reagent (Invitrogen; Thermo Fisher Scientific, Inc.) was used to extract total RNA from serum and cell samples, and the obtained RNA was reversed transcribed into cDNA using a PrimeScript RT reagent kit (Takara Bio, Inc.) according to the manufacturer's protocol. The expression of miR-328-3p and the mRNA expression of
PTEN was quantified via qPCR, which was performed using SYBR-Green I Master Mix kit (Invitrogen; Thermo Fisher Scientific, Inc.) on a 7500 Real-Time PCR System (Applied Biosystems; Thermo Fisher Scientific, Inc.). The thermocycling conditions were set as follows: $95^{\circ} \mathrm{C}$ for $10 \mathrm{~min}$, followed by 40 cycles of $95^{\circ} \mathrm{C}$ for $20 \mathrm{sec}, 58^{\circ} \mathrm{C}$ for $10 \mathrm{sec}$ and $72^{\circ} \mathrm{C}$ for $20 \mathrm{sec}$. The expression levels of miR-328-3p and PTEN were normalized to U6 and GAPDH, respectively, and calculated using the $2^{-\Delta \Delta C q}$ method (16). The following primer sequences were used in the reactions: $\mathrm{miR}-328-3 p$ forward, 5'-GCCGAG CTGGCCCTCUCTGC-3' and reverse, 5'-CTCAACTGGTGT CGTGGA-3'; PTEN forward, 5'-TCCCAGACATGACAG CCATC-3' and reverse, 5'-TGCTTTGAATCCAAAAAC CTTACT-3'; U6 forward, 5'-CTCGCTTCGGCAGCACA-3' and reverse, 5'-AACGCTTCACGAATTTGCGT-3'; GAPDH forward, 5'-CAATGACCCCTTCATTGACC-3' and reverse, 5'-TTGATTTTGGAGGGATCTCG-3'.

Cell proliferation assay. After $48 \mathrm{~h}$ of cell transfection, the proliferation of hFOB1.19 was estimated using an MTT assay. Cells $\left(3 \times 10^{3}\right.$ cells/well) were seeded into 96 -well plates and cultured at $37^{\circ} \mathrm{C}$. The proliferation of cells at $0,24,48$ and $72 \mathrm{~h}$ was measured by incubating cells with $5 \mathrm{mg} / \mathrm{ml}$ MTT solution (Sigma-Aldrich; Merck KGaA) for $4 \mathrm{~h}$ at $37^{\circ} \mathrm{C}$. Subsequently, $150 \mu \mathrm{l}$ DMSO (Sigma-Aldrich; Merck KGaA) was added into the wells to dissolve formazan for $30 \mathrm{~min}$ at $37^{\circ} \mathrm{C}$. The optical density of samples at $490 \mathrm{~nm}$ was measured using a microplate reader (Bio-Rad Laboratories, Inc.) to reflect cell proliferation.

Apoptosis assay. The apoptosis of hFOB1.19 was evaluated using an Annexin V-FITC Apoptosis Detection kit (Nanjing

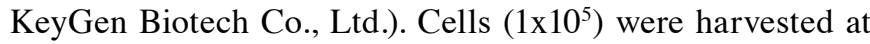
$48 \mathrm{~h}$ post-transcription and washed with PBS three times. After suspending with Annexin V binding buffer, cells were stained with $5 \mu \mathrm{l}$ V-FITC and $10 \mu \mathrm{l}$ propidium iodide for $20 \mathrm{~min}$ in the dark at room temperature. Apoptosis was measured using a flow cytometer (Attune NxT; BD Biosciences) and analyzed using the CellQuest Pro analysis software (version 5.1; BD Biosciences). Apoptotic rate, including early and late apoptosis, was calculated.

Bioinformatics prediction and luciferase reporter assay. The targets of miR-328-3p were predicted using TargetScan (http://www.targetscan.org/vert_72/), the results of which predicted that PTEN was a potential target gene of miR-328-3p. The wild-type (WT) and mutant type (MUT) 3'-UTR sequences of PTEN with a concentration of $100 \mathrm{nM}$ were synthesized by Shanghai GenePharma Co., Ltd., and were combined into the luciferase reporter vector PMIR-RB-REPORT (Guangzhou RiboBio Co., Ltd.) to perform the luciferase reporter assay. The recombinant vectors were co-transfected with miR-328-3p mimic or mimic NC using Lipofectamine ${ }^{\circledR} 3000$ (Invitrogen; Thermo Fisher Scientific, Inc.). After $48 \mathrm{~h}$ of transfection, the relative luciferase activity was detected using a Luciferase Reporter System (Promega Corporation) and normalized to Renilla luciferase activity.

Western blotting. Total protein was extracted from hFOB1.19 cells using RIPA buffer (Beyotime Institute of Biotechnology), and the concentration of proteins was determined using a 
Table I. Baseline characteristics of the participants in this study.

\begin{tabular}{|c|c|c|c|}
\hline Variables & Healthy controls $(n=40)$ & Patients with a fracture $(n=80)$ & P-value \\
\hline Age, years & $68.10 \pm 12.05$ & $68.94 \pm 11.30$ & 0.682 \\
\hline Sex & & & 0.603 \\
\hline Female, n (\%) & $21(52.5)$ & $46(57.5)$ & \\
\hline Male, $\mathrm{n}$ & $19(47.5)$ & $34(42.5)$ & \\
\hline BMI $\left(\mathrm{kg} / \mathrm{m}^{2}\right)$ & $23.03 \pm 2.81$ & $22.04 \pm 2.74$ & 0.068 \\
\hline $25 \mathrm{OHD}(\mathrm{ng} / \mathrm{ml})$ & $30.56 \pm 7.74$ & $25.91 \pm 6.64$ & $0.002^{\mathrm{a}}$ \\
\hline Osteoporosis, n (\%) & $16(40.0)$ & $63(78.8)$ & $<0.001^{\mathrm{a}}$ \\
\hline Fracture sites, n (\%) & & & - \\
\hline Vertebrae & - & $38(47.5)$ & \\
\hline Hip & - & $22(27.5)$ & \\
\hline Wrist & - & $20(25.0)$ & \\
\hline
\end{tabular}

${ }^{a} \mathrm{P}<0.05$. Data are presented as the mean \pm SD unless otherwise stated. BMI, body mass index; 25OHD, 25-hydroxyvitamin D.

bicinchoninic acid kit (Beyotime Institute of Biotechnology). Protein were separated by $10 \%$ SDS-PAGE and transferred to PVDF membranes (EMD Millipore). After blocking with $5 \%$ skimmed milk at $37^{\circ} \mathrm{C}$ for $1 \mathrm{~h}$, membranes were incubated with the following primary antibodies at $4^{\circ} \mathrm{C}$ overnight: Anti-AKT (1:1,000; cat.no.4691), anti-phosphorylated (p)-AKT (1:2,000; cat. no. 4060), anti-PTEN (1:1,000; cat. no. 9188) and anti-GAPDH (1:1,000; cat. no. 5174; all purchased from Cell Signaling Technology, Inc.). Membranes were then incubated with horseradish peroxide-labelled anti-rabbit IgG secondary antibodies (1:1,000; cat. no. 7074; Cell Signaling Technology, Inc.). The protein bands were visualized by enhanced chemiluminescence (Thermo Fisher Scientific, Inc.) and GAPDH was used as a loading control. Protein bands were quantified using ImageJ software (1.46; National Institutes of Health).

Statistical analysis. The data analyzed in the present study were presented as the mean \pm SD. Each experiment was repeated at least three times. Differences between two groups were analyzed using unpaired Student's t-test or $\chi^{2}$ test, and comparison between multiple groups was assessed using one-way ANOVA followed by Tukey's post hoc test. The Pearson correlation coefficient test was used to evaluate correlation. SPSS 22.0 (IBM Corp.) and GraphPad Prism 7.0 software (GraphPad Software, Inc.) were used to perform analyses. $\mathrm{P}<0.05$ was considered to indicate a statistically significant difference.

\section{Results}

Baseline characteristics of the participants. The age, sex, body mass index (BMI) and 25-hydroxyvitamine D (25OHD) of patients with a fracture and healthy controls were listed and compared in Table I. The 80 patients with a fracture included $38(47.5 \%)$ vertebral fracture cases, 22 (27.5\%) hip fracture cases and $20(25.0 \%)$ wrist fracture cases. No significant differences were observed between the age, sex and BMI of the two groups $(\mathrm{P}>0.05)$. Patients with a fracture had significantly lower 25OHD levels and higher incidence rates of osteoporosis compared with healthy controls $(\mathrm{P}<0.01)$.

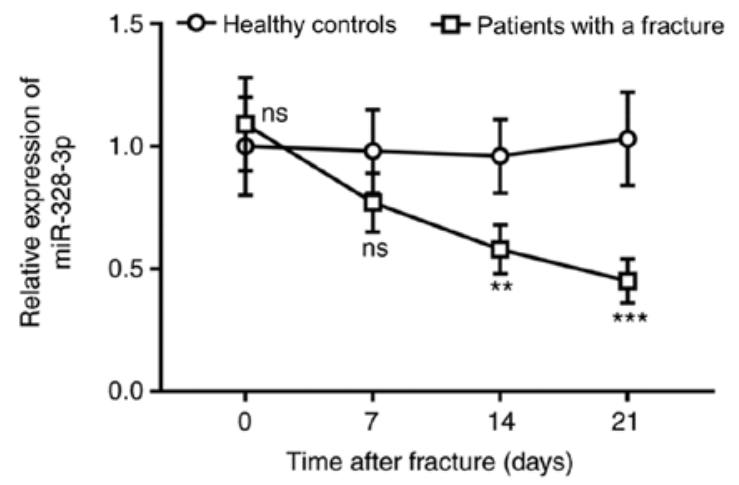

Figure 1. Dynamic serum expression of miR-328-3p in patients with fracture. ${ }^{* *} \mathrm{P}<0.01$ and ${ }^{* * *} \mathrm{P}<0.001$ vs. healthy controls. ns, not significant; miR, microRNA.

Dynamic expression patterns of miR-328-3p in patients with fracture. The serum expression levels of miR-328-3p were slightly higher in patients with fracture compared with those of healthy controls $\leq 24 \mathrm{~h}$ post fracture and before fixation therapy ( $\mathrm{P}>0.05$; Fig. 1). After fixation, no significant differences were observed in the serum expression levels of miR-328-3p between the patients with a fracture and healthy controls for 7 days $(\mathrm{P}>0.05)$. After that, miR-328-3p expression levels gradually decreased and were significantly downregulated on day 14 and 21 in patients with fracture compared with healthy individuals $(\mathrm{P}<0.01$; Fig. 1$)$. The results suggested that miR-328-3p may be involved in the progression of fracture healing.

Effects of miR-328-3p on osteoblastic proliferation and apoptosis. To understand the mechanisms underlying the aberrant expression of miR-328-3p during the healing process of patients with fracture, the effect of miR-328-3p on osteoblastic viability was investigated. The expression levels of miR-328-3p in hFOB1.19 were successfully overexpressed by the miR-328-3p mimic $(\mathrm{P}<0.001$; Fig. $2 \mathrm{~A})$. The proliferation of osteoblasts was significantly increased by the overexpression of miR-328-3p ( $\mathrm{P}<0.05$; Fig. 2B). Additionally, 

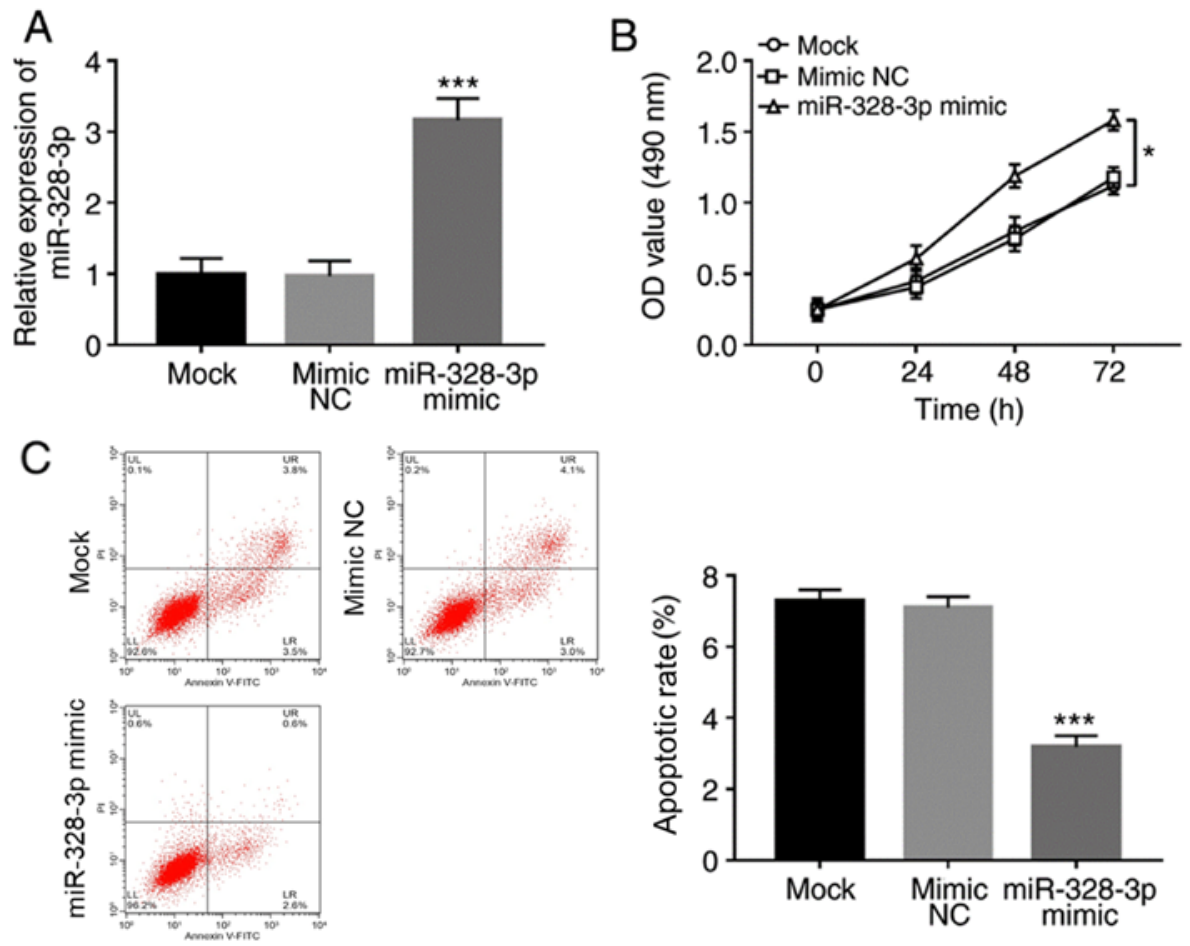

Figure 2. Effect of miR-328-3p on osteoblastic proliferation and apoptosis of hFOB1.19. (A) Expression of miR-328-3p was overexpressed by miR-328-3p mimic. (B) Overexpression of miR-328-3p led to enhanced osteoblastic proliferation. (C) The apoptosis of hFOB1.19 cells was inhibited by the overexpression of miR-328-3p. ${ }^{*} \mathrm{P}<0.05$ and ${ }^{* * *} \mathrm{P}<0.001$ vs. mimic NC. miR, microRNA; OD, optical density; NC, negative control.
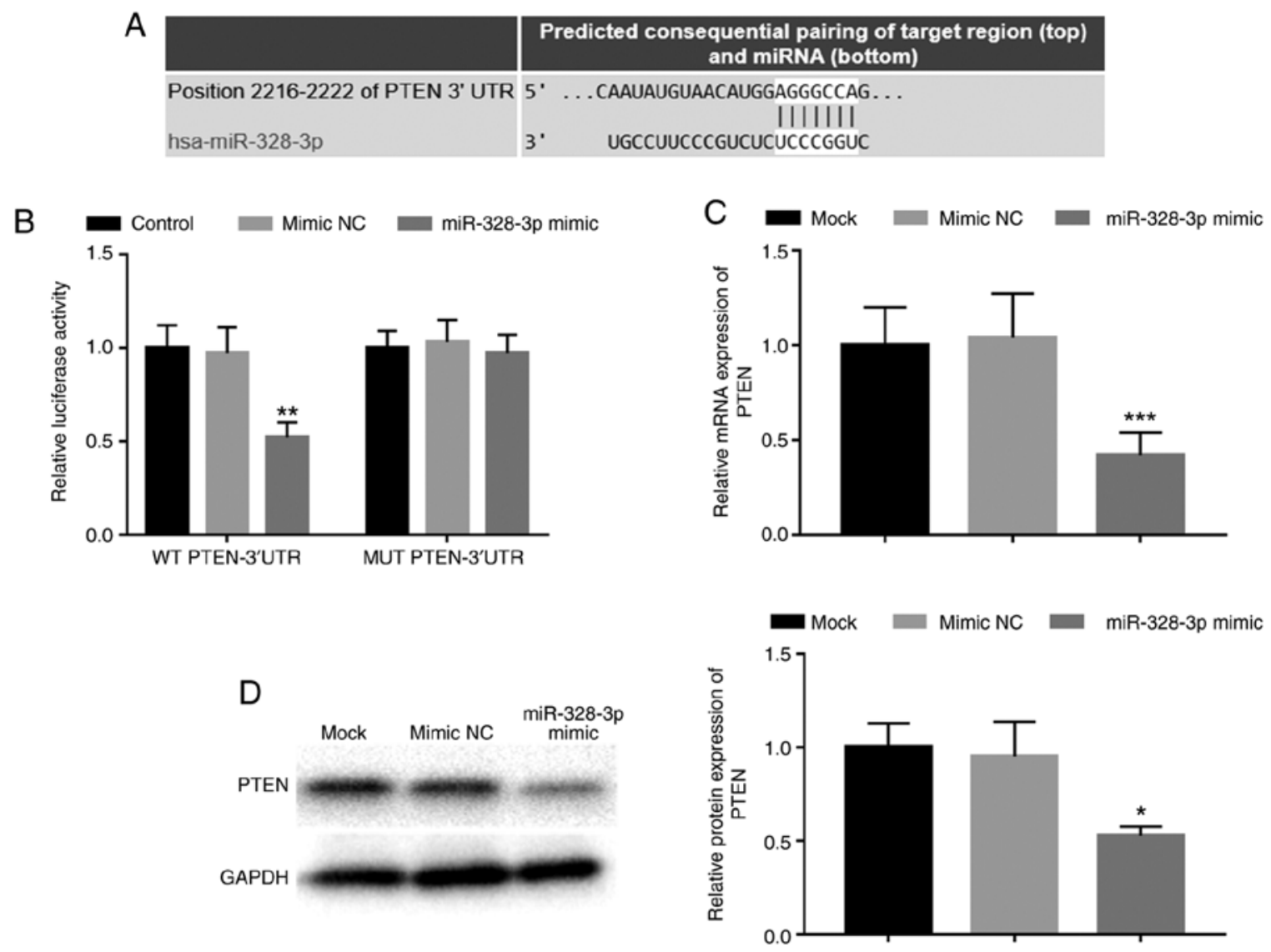

Figure 3. miR-328-3p directly inhibits the expression of PTEN in osteoblasts. (A) The putative binding site of miR-328-3p at the 3'-UTR of PTEN. (B) The relative luciferase activity was inhibited by the overexpression of miR-328-3p in WT PTEN-3'UTR group. (C) The mRNA expression of PTEN was significantly reduced in hFOB1.19 cells with miR-328-3p overexpression. (D) The protein expression of PTEN was also decreased by miR-328-3p overexpression in hFOB1.19 cells. ${ }^{*} \mathrm{P}<0.05,{ }^{* *} \mathrm{P}<0.01$ and $^{* * * *} \mathrm{P}<0.001$ vs. mimic NC. miR, microRNA; UTR, untranslated region; WT, wild-type; MUT, mutated; NC, negative control. 

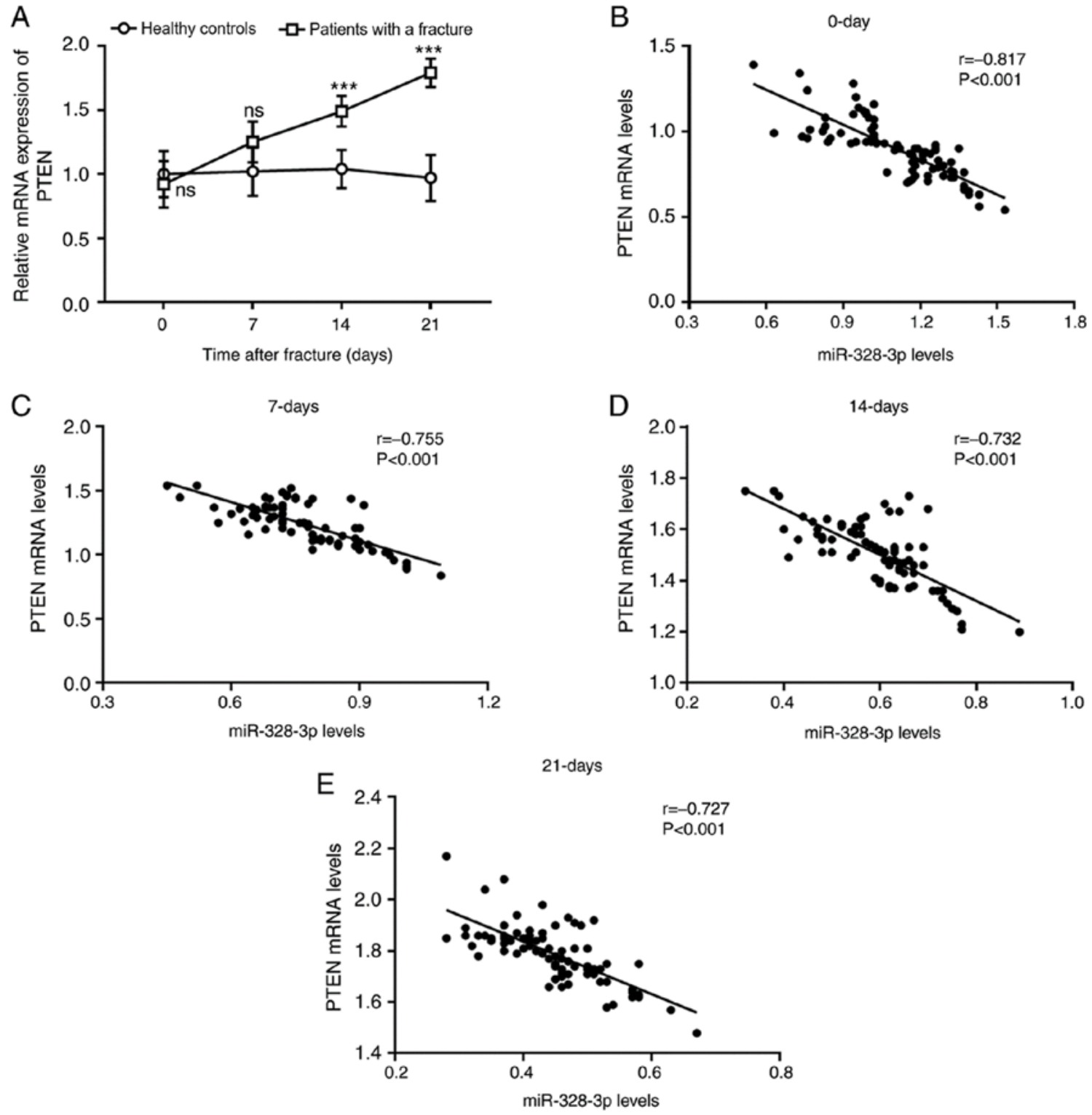

Figure 4. Correlation of miR-328-3p serums levels and PTEN in patients with fracture. (A) Dynamic expression of PTEN in patients with fracture. Serum miR-328-3p expression levels were negatively correlated with PTEN expression in patients (B) before fixation (0) and (C) 7, (D) 14 and (E) 21 days post fixation therapy. ${ }^{* * *} \mathrm{P}<0.001$ vs. healthy controls. miR, microRNA; ns, not significant.

apoptotic rate was significantly reduced in cells transfected with miR-328-3p mimic compared with cells transfected with mimic $\mathrm{NC}(\mathrm{P}<0.001$; Fig. $2 \mathrm{C})$.

miR-328-3p directly regulates PTEN in osteoblasts. Bioinformatics prediction revealed a putative binding site of miR-328-3p at the 3'-UTR of PTEN (Fig. 3A). Thus, a luciferase reporter assay was performed to confirm the relationship between miR-328-3p and PTEN. The results demonstrated that the relative luciferase activity in the WT PTEN-3'UTR group was significantly inhibited by the overexpression of miR-328-3p when compared with cells co-transfected with mimic NC $(\mathrm{P}<0.01$; Fig. 3B), whereas no significant differences were observed in the MUT PTEN-3'UTR group ( $\mathrm{P}>0.05$; Fig. 3B). Additionally, the regulatory effect of miR-328-3p on PTEN in osteoblasts was examined. The results demonstrated that overexpression of miR-328-3p expression significantly decreased the mRNA and protein expression levels of PTEN compared with those of the mimic NC group in hFOB1.19 cells $(\mathrm{P}<0.05$; Fig. 3B and $\mathrm{C})$.

Correlation between miR-328-3p and PTEN in patients with fracture. The mRNA expression levels of PTEN in patients with fracture were evaluated. No significant differences were observed in the expression levels of PTEN between patients with fracture and healthy controls before fixation therapy and within 7 days post therapy $(\mathrm{P}>0.05)$. However, the expression levels PTEN were significantly increased on days 14 and 21 day in patients with fracture compared with healthy controls $(\mathrm{P}<0.001$; Fig. 4A). Additionally, the correlation of serum miR-328-3p and PTEN levels was analyzed; at each time point, a negative correlation was observed between serum levels of miR-328-3p and PTEN (P<0.001; Fig. 4B-E).

PTEN mediates the regulatory effects of miR-328-3p on osteoblastic viability. PTEN is known to serve pivotal roles in the 
A

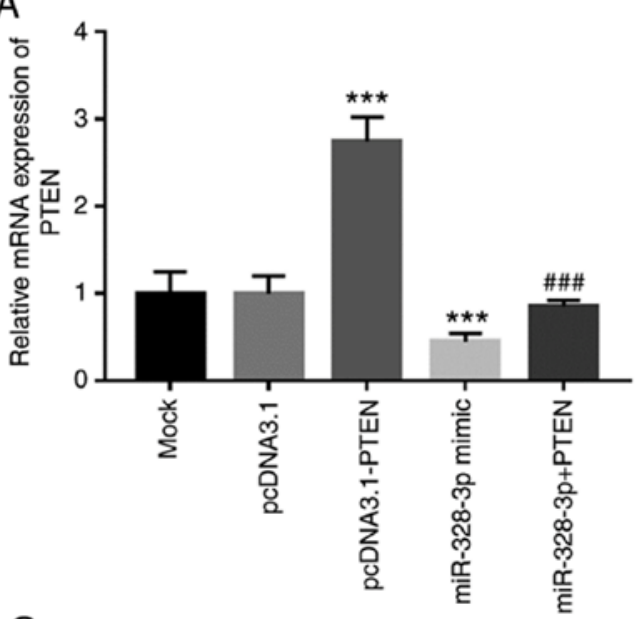

C
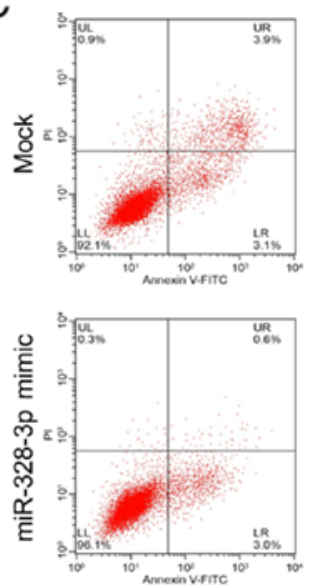
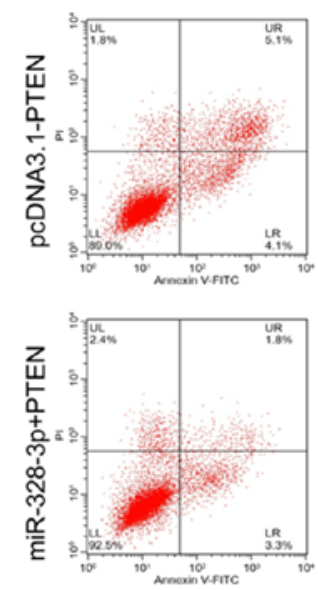

B
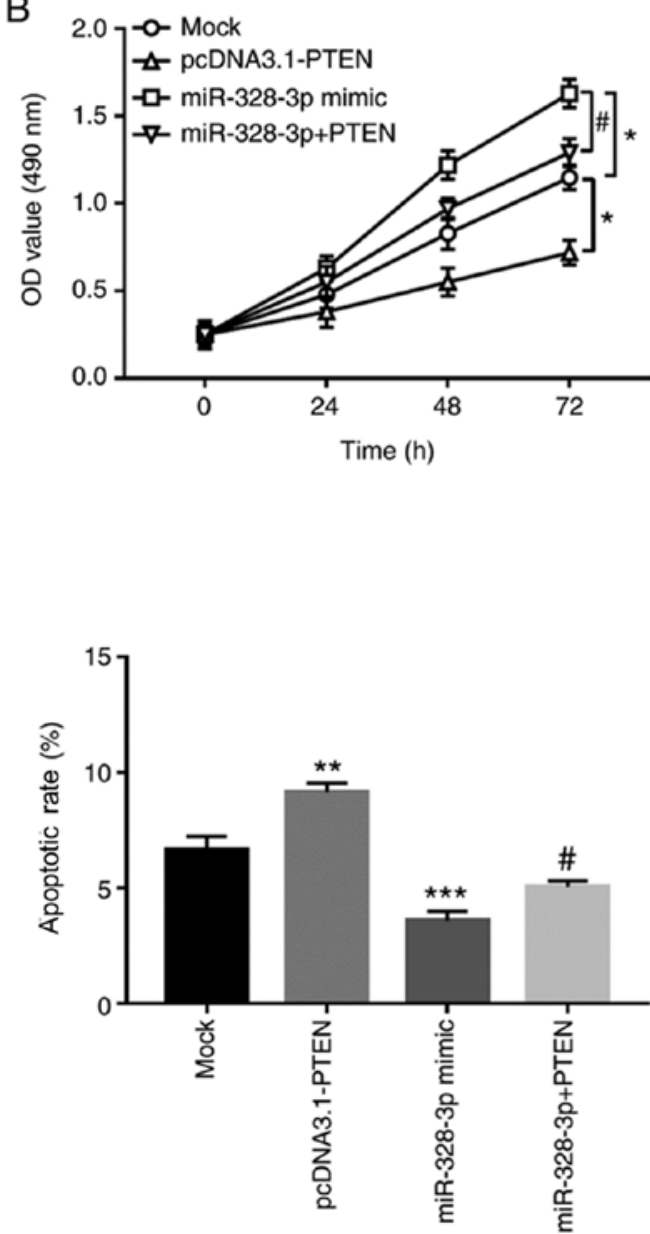

Figure 5. miR-328-3p regulates osteoblastic viability by targeting PTEN. (A) pcDNA3.1-PTEN-induced overexpression of PTEN in hFOB1.19 cells and reversed the effect of miR-328-3p overexpression on PTEN expression. (B) PTEN overexpression inhibited osteoblastic proliferation as well as the miR-328-3p mimic-induced increased cell proliferation. (C) Osteoblastic apoptosis was increased in cells with PTEN reduction and the inhibited cell apoptosis caused by miR-328-3p overexpression was abolished by the overexpression of $\mathrm{PTEN}$. ${ }^{*} \mathrm{P}<0.05,{ }^{* *} \mathrm{P}<0.01$ and ${ }^{* * * *} \mathrm{P}<0.001 \mathrm{vs}$. mock; ${ }^{*} \mathrm{P}<0.05$ and ${ }^{\# \# \#} \mathrm{P}<0.05$ vs. miR-328-3p mimic. miR, microRNA.

regulation of a cells biological function (17), and the inhibition of PTEN has been reported to promote long non-coding RNA GHET1 on osteoblast proliferation and differentiation (18). Thus, the effect of miR-328-3p on osteoblastic viability may be mediated by PTEN. pcDNA3.1-PTEN was used to overexpress PTEN in hFOB1.19 cells (Fig. 5A). Overexpression of PTEN significantly inhibited osteoblastic proliferation, but significantly increased osteoblastic apoptosis $(\mathrm{P}<0.05$; Fig. 5B and $\mathrm{C})$. Additionally, in cells co-transfected with miR-328-3p mimic and pcDNA3.1-PTEN, the expression levels of PTEN were significantly reduced compared with those of cells transfected with pcDNA3.1-PTEN alone ( $<<0.001$; Fig. 5A). Notably, overexpression of PTEN by pcDNA3.1-PTEN significantly reversed the effect of miR-328-3p on osteoblastic viability, which was demonstrated by the inhibited cell proliferation and enhanced apoptosis in hFOB1.19 ( $\mathrm{P}<0.05$; Fig. 5B and C).

Regulatory effect of miR-328-3p on the PI3K/AKT signaling pathway. PTEN is a negative regulator of the PI3K/AKT signaling pathway, which is one of the most important pathways during the processes of cell proliferation and apoptosis (19). Thus, the present study further explored the effect of miR-328-3p on the activity of PI3K/AKT signaling. The results demonstrated that the ratio of p-AKT/AKT was significantly increased in hFOB1.19 cells with overexpression of miR-328-3p when compared with the mock group $(\mathrm{P}<0.001$; Fig. 6), which suggested that the activity of the AKT signaling pathway was enhanced by the overexpression of miR-328-3p. Additionally, in the cells overexpressing PTEN, the increase in $\mathrm{p}-\mathrm{AKT} / \mathrm{AKT}$ ratio induced by $\mathrm{miR}-328-3 \mathrm{p}$ overexpression was significantly attenuated $(\mathrm{P}<0.01$; Fig. 6), suggesting that miR-328-3p may activate the PI3K/AKT signaling by inhibiting PTEN.

\section{Discussion}

Bone regeneration is the key to fracture healing, which involves the proliferation, apoptosis and differentiation of osteoblasts (20), implying that methods that regulate osteoblastic activity may assist fracture healing. miRNAs have important regulatory effects on various cellular processes, such as cell proliferation, migration, invasion, differentiation and apoptosis (21). Emerging studies have focused on deregulated miRNAs during fracture healing, which are expected 


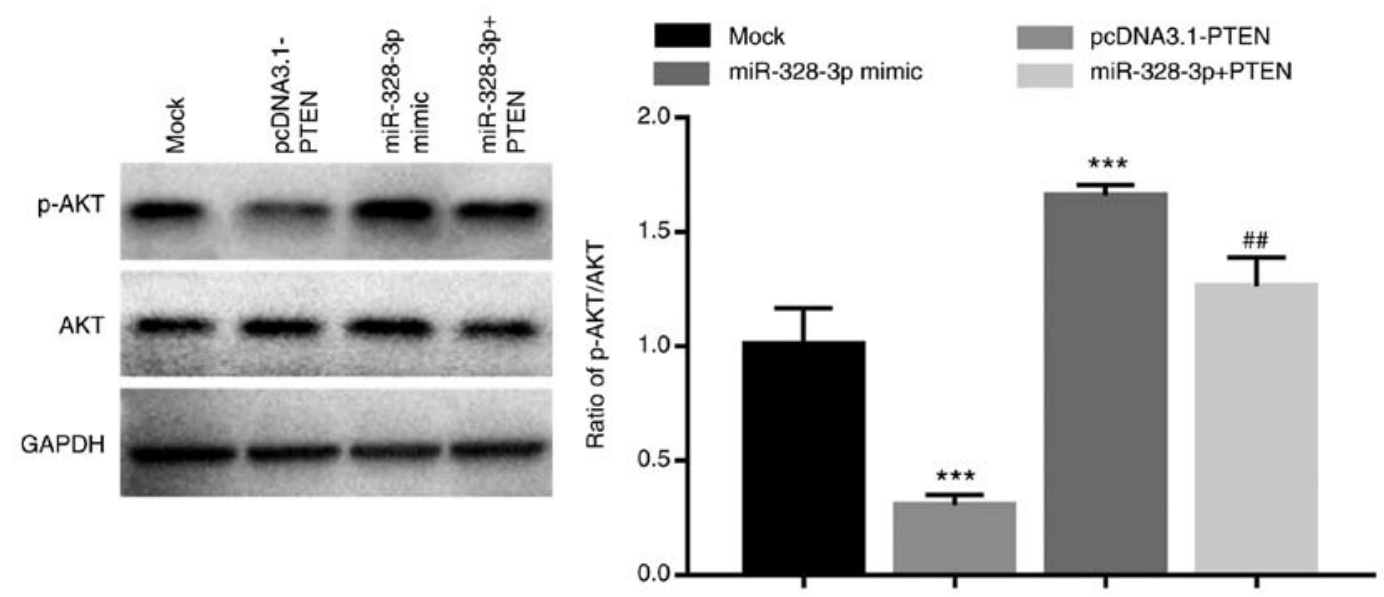

Figure 6. Effect of miR-328-3p and PTEN on the activation of PI3K/AKT signaling pathway. The ratio of p-AKT/AKT was enhanced by the overexpression of miR-328-3p. ${ }^{* * *} \mathrm{P}<0.001$ vs. mock; ${ }^{* \#} \mathrm{P}<0.01$ vs. miR-328-3p mimic. miR, microRNA; $\mathrm{p}$, phosphorylated.

to promote the development of novel strategies to accelerate healing by improving osteoblastic viability. For example, Lang et al (22) provided evidence for the use of miR-25 to promote fracture healing by enhancing osteoblastic proliferation and differentiation through activation of the Wnt signaling pathway. Mi et al (23) demonstrated that decreased miR-7223-5p during fracture healing regulated osteoblastic proliferation and apoptosis by targeting PI3KR1, mediating the promoting effect of circRNA AFF4 on fracture healing. The expression of miR-203 in patients with fractures was also aberrant during healing and could inhibit the proliferation and promote the apoptosis of osteoblast cells by targeting prostate and breast cancer overexpressed 1 (24). These data indicate the important roles of miRNAs, which have regulatory effects on osteoblastic viability in fracture healing.

A previous study on differentially expressed miRNAs in patients with osteoporosis reported that patients with osteoporosis who develop fractures had significantly decreased miR-328-3p levels (15), suggesting that miR-328-3p may be associated with bone regeneration and progression of osteoporosis. There is some evidence that miR-328-3p regulates cell proliferation and apoptosis; the decreased expression of miR-328-3p in patients with osteosarcoma has been reported to be involved in tumor progression by regulating osteosarcoma cell proliferation, migration and apoptosis (25). The expression of miR-328-3p in hepatocellular carcinoma cells was also demonstrated to be reduced and could decrease tumor cell proliferation and increase apoptosis (26). However, the relationship between miR-328-3p and osteoblastic viability has rarely been reported. In the present study, the serum expression levels of miR-328-3p was demonstrated to be slightly higher in patients with fracture before fixation treatment and exhibited no significant changes 7 days post fixation. However, its expression was markedly decreased 14 and 21 days after fixation. The abnormal expression patterns of miR-328-3p suggested its role during fracture healing. Osteoporosis is the most frequent underlying cause of fragility fracture (27). A total of $78.8 \%$ of the patients with fracture enrolled in the current study had osteoporosis, and the majority of female patients were postmenopausal women, who have high risk of osteoporosis (28). Circulating miR-328-3p has been identified as a miRNA associated with osteoblast differentiation in osteoporosis (29), which also indicated the crucial role of miR-328-3p in osteoporosis pathogenesis and progression. The present study demonstrated that the overexpression of miR-328-3p markedly increased osteoblastic proliferation but decreased cell apoptosis, indicating the important regulatory effect of miR-328-3p on osteoblastic viability. Considering the strong demand for enough osteoblasts for fracture healing, the present study hypothesized that the slight increase in miR-328-3p at an early time of fracture is required to obtain a sufficient number of osteoblasts. After the accumulation of osteoblasts, the expression of miR-328-3p was downregulated, the proliferation of osteoblasts was decreased and the inhibition of apoptosis was attenuated. At the later stage of fracture healing, miR-328-3p expression was significantly reduced, leading to the osteoblasts proliferating slowly but further differentiating into mature bone cells and chondrocytes, thereby achieving bone regeneration. Therefore, the methods to increase miR-328-3p may reduce the time spent in the early stage of fracture healing by promoting osteoblastic viability.

According to bioinformatics prediction, PTEN was identified as a target gene of miR-328-3p, and its interaction with miR-328-3p was demonstrated using a luciferase reporter assay. PTEN is a key molecule involved in various cellular processes $(30,31)$. For example, PTEN acted as a target gene of miR-29a-3p and could mediate the regulatory effects of miR-29a-39 on vascular smooth muscle cell proliferation and apoptosis (30). Another example demonstrated that the regulatory role of methyltransferase-like protein 3 (METTL3) regulated retinal pigment epithelium cell proliferation, apoptosis and pyroptosis by inhibiting PTEN (31). In addition, Ge et al (32) reported that PTEN inhibited fracture healing by suppressing osteogenic differentiation of mesenchymal stem cells. Mice with PTEN knockdown in osteoblasts demonstrated significantly improved fracture healing (33). The present study demonstrated lower PTEN expression levels at the early stage of fracture, but its expression was significantly increased 14 days post fixation, which was negatively correlated with serum miR-328-3p levels in patients with fracture. The results suggested that the inhibition of PTEN was required for the rapid proliferation of osteoblasts for fracture healing. 
Furthermore, the overexpression of miR-328-3p inhibited PTEN expression in osteoblasts, and PTEN overexpression reversed the effect of miR-328-3p on osteoblastic proliferation and apoptosis, which suggested that miR-328-3p may promote osteoblastic viability by targeting PTEN.

The PI3K/AKT pathway is a well-known signaling pathway that is important in regulating cell proliferation, cell cycle progression and apoptosis, and its beneficial role in fracture healing has been well reported $(34,35)$. PTEN acts as a major inhibitor of this signaling pathway, thereby participating in various cellular processes (36). In the osteoblasts overexpressing miR-328-3p, the expression of p-AKT was elevated, suggesting that the overexpression of miR-328-3p promoted the activation of the PI3K/AKT signaling pathway. Furthermore, elevated p-AKT induced by miR-328-3p was abolished by the overexpression of PTEN, which implied that the regulatory effect of miR-328-3p on the PI3K/AKT signaling may be mediated by PTEN. Collectively, miR-328-3p may facilitate osteoblastic viability by activating the PI3K/AKT signaling pathway via PTEN.

The present study may provide novel insights into the pathogenesis of fracture healing, and the methods to increase miR-328-3p expression may be used to shorten healing time in patients with fracture. However, the present study lacks animal experiments, and further in vivo studies are required. Another limitation of the present study is that the osteoblastic viability under the inhibition of miR-328-3p was not assessed. Thus, future studies should include in vitro functional experiments using both miR-328-3p mimics and inhibitors. In addition, fracture healing involves complex biological processes and although the pesent study demonstrated the regulatory effect of miR-328-3p on osteoblastic viability, further studies are necessary to confirm the role of miR-328-3p in fracture healing and the underlying mechanisms by considering other aspects that are associated with bone repair, such as osteoblast differentiation, bone matrix protein production and mineralization. In conclusion, the present study suggested that the aberrant expression of miR-328-3p in patients with a fracture may promote fracture healing by accelerating osteoblastic viability through the PTEN/PI3K/AKT pathway.

\section{Acknowledgements}

Not applicable.

\section{Funding}

No funding was received.

\section{Availability of data and materials}

All data generated or analyzed during this study are included in this published article.

\section{Authors' contributions}

WX and ZZ contributed to the study design, the data collection, data interpretation and manuscript preparation. $\mathrm{ZW}$ and YZ contributed to data collection, data analysis and statistical analysis. All authors read and approved the final manuscript.

\section{Ethics approval and consent to participate}

The present study was approved by the Ethics Committee of Traditional Chinese Medicine Hospital of Weifang and written informed consent was obtained from each patient.

\section{Patient consent for publication}

Written informed consent for publication was obtained from each participant.

\section{Competing interests}

The authors declare that they have no competing interests.

\section{References}

1. Dreinhofer KE, Mitchell PJ, Bégué T, Cooper C, Costa ML, Falaschi P, Hertz K, Marsh D, Maggi S, Nana A, et al: A global call to action to improve the care of people with fragility fractures. Injury 49: 1393-1397, 2018.

2. Horowitz JA, Puvanesarajah V, Jain A, Raad M, Gjolaj JP, Shen FH and Hassanzadeh H: Fragility fracture risk in elderly patients with cervical myelopathy. Spine (Phila Pa 1976) 44: 96-102, 2019.

3. Friedman SM and Mendelson DA: Epidemiology of fragility fractures. Clin Geriatr Med 30: 175-181, 2014.

4. Wasserman H, Backeljauw PF, Khoury JC, Kalkwarf HJ and Gordon CM: Bone fragility in Turner syndrome: Fracture prevalence and risk factors determined by a national patient survey. Clin Endocrinol (Oxf) 89: 46-55, 2018.

5. Oryan A, Monazzah S and Bigham-Sadegh A: Bone injury and fracture healing biology. Biomed Environ Sci 28: 57-71, 2015.

6. Cheng C and Shoback D: Mechanisms underlying normal fracture healing and risk factors for delayed healing. Curr Osteoporos Rep 17: 36-47, 2019.

7. Mohr AM and Mott JL: Overview of microRNA biology. Semin Liver Dis 35: 3-11, 2015.

8. Wang CZ, Deng F, Li H, Wang DD, Zhang W, Ding L and Tang JH: MiR-101: A potential therapeutic target of cancers. Am J Transl Res 10: 3310-3321, 2018.

9. Zhang B, Yi J, Zhang CL, Zhang QH, Xu JF, Shen HQ and Ge DW: MiR-146a inhibits proliferation and induces apoptosis in murine osteoblastic MC3T3-E1 by regulating Bcl2. Eur Rev Med Pharmacol Sci 21: 3754-3762, 2017.

10. Li X, Li K, Yu G, Yu C and Liu C: miR-342-5p inhibits expression of Bmp7 to regulate proliferation, differentiation and migration of osteoblasts. Mol Immunol 114: 251-259, 2019.

11. Tu XM, Gu YL and Ren GQ: miR-125a-3p targetedly regulates GIT1 expression to inhibit osteoblastic proliferation and differentiation. Exp Ther Med 12: 4099-4106, 2016.

12. Hu WX, Li H and Jiang JZ: MiR-491-3p is down-regulated in postmenopausal osteoporosis and affects growth, differentiation and apoptosis of hFOB1.19 cells through targeting CTSS. Folia Histochem Cytobiol 1: 9-16, 2020.

13. Pan S, Ren F, Li L, Liu D, Li Y, Wang A, Li W, Dong Y and Guo W: MiR-328-3p inhibits cell proliferation and metastasis in colorectal cancer by targeting Girdin and inhibiting the PI3K/Akt signaling pathway. Exp Cell Res 390: 111939, 2020.

14. Yi W, Tu MJ, Liu Z, Zhang C, Batra N, Yu AX and Yu AM: Bioengineered miR-328-3p modulates GLUT1-mediated glucose uptake and metabolism to exert synergistic antiproliferative effects with chemotherapeutics. Acta Pharm Sin B 10: 159-170, 2020.

15. Weilner S, Skalicky S, Salzer B, Keider V, Wagner M, Hildner F, Gabriel C, Dovjak P, Pietschmann P, Grillari-Voglauer R, et al: Differentially circulating miRNAs after recent osteoporotic fractures can influence osteogenic differentiation. Bone 79: 43-51, 2015.

16. Livak KJ and Schmittgen TD: Analysis of relative gene expression data using real-time quantitative PCR and the 2(-Delta Delta C(T)) method. Methods 25: 402-408, 2001.

17. Malaney P, Uversky VN and Dave V: PTEN proteoforms in biology and disease. Cell Mol Life Sci 74: 2783-2794, 2017. 
18. Li D, Li L, Chen X, Gao Y, Cao Y and Hao B: LncRNA GHET1 promotes osteoblast proliferation and differentiation by inhibiting PTEN. Panminerva Med Jul 29, 2019 (Online ahead of print).

19. Xu W, Yang Z, Xie C, Zhu Y, Shu X, Zhang Z, Li N, Chai N, Zhang S, Wu K, et al: PTEN lipid phosphatase inactivation links the hippo and PI3K/Akt pathways to induce gastric tumorigenesis. J Exp Clin Cancer Res 37: 198, 2018.

20. Einhorn TA and Gerstenfeld LC: Fracture healing: Mechanisms and interventions. Nat Rev Rheumatol 11: 45-54, 2015.

21. Zhao C, Zhou Y, Ran Q, Yao Y, Zhang H, Ju J, Yang T, Zhang W, Yu X and He S: MicroRNA-381-3p functions as a dual suppressor of apoptosis and necroptosis and promotes proliferation of renal cancer cells. Front Cell Dev Biol 8: 290, 2020.

22. Lang Y, Sun Q, Zhu LM, Qiu XD, Hu BS, Yang J and Zhang JD: MiR-25 overexpression promotes fracture healing by activating the Wnt signaling pathway. Eur Rev Med Pharmacol Sci 23: 7200-7208, 2019.

23. Mi B, Xiong Y, Chen L, Yan C, Endo Y, Liu Y, Liu J, Hu L, $\mathrm{Hu}$ Y, Sun Y, et al: CircRNA AFF4 promotes osteoblast cells proliferation and inhibits apoptosis via the Mir-7223-5p/PIK3R1 axis. Aging (Albany NY) 11: 11988-12001, 2019.

24. Zhang SY, Gao F, Peng CG, Zheng CJ and Wu MF: Hsa-miR-203 inhibits fracture healing via targeting PBOV1. Eur Rev Med Pharmacol Sci 22: 5797-5803, 2018.

25. Shi J, An G, Guan Y, Wei T, Peng Z, Liang M and Wang Y: miR-328-3p mediates the anti-tumor effect in osteosarcoma via directly targeting MMP-16. Cancer Cell Int 19: 104, 2019.

26. Li JZ, Li J and Liu BZ: MicroRNA-328-3p inhibits malignant progression of hepatocellular carcinoma by regulating MMP-9 level. Eur Rev Med Pharmacol Sci 23: 9331-9340, 2019.

27. Rommens PM, Wagner D and Hofmann A: Fragility fractures of the pelvis. JBJS Rev 5: 01874474-201703000-00004, 2017.

28. Rossi LMM, Copes RM, Dal Osto LC, Flores C, Comim FV and Premaor MO: Factors related with osteoporosis treatment in postmenopausal women. Medicine (Baltimore) 97: e11524, 2018.

29. Chen R, Liao X, Chen F, Wang B, Huang J, Jian G, Huang Z, Yin G, Liu H and Jin D: Circulating microRNAs, miR-10b-5p, miR-328-3p, miR-100 and let-7, are associated with osteoblast differentiation in osteoporosis. Int J Clin Exp Pathol 11: 1383-1390, 2018
30. Zhou Y, Wang M, Zhang J, Xu P and Wang H: MicroRNA-29a-3p regulates abdominal aortic aneurysm development and progression via direct interaction with PTEN. J Cell Physiol 235: 9414-9423, 2020.

31. Zha X, Xi X, Fan X, Ma M, Zhang Y and Yang Y: Overexpression of METTL3 attenuates high-glucose induced RPE cell pyroptosis by regulating miR-25-3p/PTEN/Akt signaling cascade through DGCR8. Aging (Albany NY) 12: 8137-8150, 2020.

32. Ge JB, Lin JT, Hong HY, Sun YJ, Li Y and Zhang CM: MiR-374b promotes osteogenic differentiation of MSCs by degrading PTEN and promoting fracture healing. Eur Rev Med Pharmacol Sci 22: 3303-3310, 2018

33. Burgers TA, Hoffmann MF, Collins CJ, Zahatnansky J, Alvarado MA, Morris MR, Sietsema DL, Mason JJ, Jones CB, Ploeg HL and Williams BO: Mice lacking pten in osteoblasts have improved intramembranous and late endochondral fracture healing. PLoS One 8: e63857, 2013.

34. Liu Y, Liu J, Xia T, Mi BB, Xiong Y, Hu LC, Ruan TY, Zhou W and Liu GH: MiR-21 promotes fracture healing by activating the PI3K/Akt signaling pathway. Eur Rev Med Pharmacol Sci 23: 2727-2733, 2019.

35. Sun Z, Liu F, Cai X, Yu W, Xu L and Yang B: MiR-126 affects femoral fracture healing in rats through PI3K/AKT signaling pathway. Panminerva Med Jun 28, 2019.

36. Yao LZ, Zhu YL and Liu JJ: Inhibition of PTEN gene expression by small interfering RNA on PI3K/Akt/FoxO3a signaling pathway in human nasopharyngeal carcinoma. Technol Cancer Res Treat 19: 1533033820917959, 2020.

This work is licensed under a Creative Commons Attribution-NonCommercial-NoDerivatives 4.0 International (CC BY-NC-ND 4.0) License. 